\title{
Differential Muscarinic Modulation of Synaptic Transmission in Dorsal and Ventral Regions of the Rat Nucleus Accumbens Core
}

\author{
X. JIANG ${ }^{1,3^{*}}$, J. J. ZHANG ${ }^{1^{*}}$, M. Y. WANG ${ }^{2}$, N. SUI ${ }^{1}$ \\ ${ }^{*}$ These authors contributed equally to this work.
}

${ }^{1}$ Key Laboratory of Mental Health, Institute of Psychology, Chinese Academy of Sciences, Beijing, China, ${ }^{2}$ Cell Electrophysiology Laboratory, Wannan Medical College, Wuhu, Anhui, China, ${ }^{3}$ University of Chinese Academy of Sciences, Beijing, China

Received January 24, 2013

Accepted August 8, 2013

On-line November 1, 2013

\section{Summary}

The nucleus accumbens (NAc) core is critical in the control of motivated behaviors. The muscarinic acetylcholine receptors (mAChRs) modulating the excitatory inputs into the NAc core have been reported to impact such behaviors. Recent studies suggest that ventral and dorsal regions of the NAc core seem to be innervated by distinct populations of glutamatergic projection neurons. To further examine mAChRs modulation of these glutamatergic inputs to the NAc core, we employed intracellular recordings in rat NAC coronal slice preparation to characterize: 1) the effects of muscarine, an mAChRs agonist, on membrane properties of the NAC core neurons; 2) depolarizing synaptic potentials (DPSP) elicited by ventral and dorsal focal electrical stimuli; and 3) paired-pulse response with paired-pulse stimulation. Here we report that the paired-pulse ratio (PPR) elicited by dorsal stimuli was greater than that elicited by ventral stimuli. Bath application of muscarine (1-30 $\mu \mathrm{M})$ decreased both ventral and dorsal DPSP in a concentration-dependent manner, with no effect on electrophysiological properties of NAc core neurons. Muscarine at $30 \mu \mathrm{M}$ also elicited larger depression of dorsal DPSP than ventral DPSP. Moreover, muscarine increased the PPR of both dorsal and ventral DPSP. These data indicate that the glutamatergic afferent fibers traversing the dorsal and ventral NAc are separate, and that differential decrease of distinct afferent excitatory neurotransmission onto NAc core neurons may be mediated by presynaptic mechanisms.

\section{Key words}

Muscarine - Nucleus accumbens - Postsynaptic potentials • Paired-pulse ratio $\bullet$ In vitro

\section{Corresponding author}

N. Sui, Mental Health Key Laboratory, Institute of Psychology, CAS, P.O. Box 1603, Beijing, 100101, China. Fax: +86-1064872070. E-mail: suin@psych.ac.cn and

M. Y. Wang, Cell Electrophysiology Laboratory, Wannan Medical College, Wuhu, Anhui, 241002, China. Fax: +86-553-3932589. E-mail: wangmy@wnmc.edu.cn

\section{Introduction}

The nucleus accumbens (NAc) plays a vital role in the generation of motivated behaviors (Berridge 2007) and is generally divided into core and shell subregions based primarily on immunohistochemical characteristics (Zahm 1999). The NAc core is thought to translate limbic sensory information, including reward-related stimuli, the context-dependent cues, into motor output (Meredith et al. 2008), forming part of a network crucial for appetitive instrumental learning implicated in drug addiction and certain psychiatric diseases (Kelley 2004, Shirayama and Chaki 2006).

The functions of NAc core are regulated by glutamate transmission (Kalivas et al. 2009, LaLumiere and Kalivas 2008). Glutamatergic inputs onto the NAc core primarily arise from basolateral amygdala (BLA), prefrontal cortex (PFC), hippocampus and thalamus. Recent studies suggest that distinct subregions of the NAc core are innervated by different glutamatergic projections (Britt et al. 2012, Humphries and Prescott 2010, Voorn et al. 2004). Neuroanatomical studies have 
demonstrated that dorsal agranular insular cortex and dorsal prelimbic cortex project primarily to the dorsal NAc core, while the ventral NAc core is mainly innervated by pathways originating in the ventral prelimbic cortex, ventral agranular insular cortex and olfactory tubercle (Groenewegen et al. 1999, Stuber et al. 2011, Voorn et al. 2004, Yuan et al. 1992). These heterogeneously distributed afferents generate distinct physiological and behavioral responses when activated selectively (Britt et al. 2012, Goto and Grace 2005, Stuber et al. 2012) and may be differently regulated by the interneurons, for example, the cholinergic interneurons, in the NAc core.

Acetylcholine (ACh) transmission by local cholinergic interneurons is widespread in the NAc. ACh regulates glutamatergic synaptic transmission in the NAc and has a critical role in NAc-mediated behavior (Crespo et al. 2006, Hikida et al. 2003, Pratt and Kelley 2004). $\mathrm{ACh}$ inhibits the release of neurotransmitter by activating presynaptic muscarinic acetylcholine receptors (mAChRs) (Pennartz and Lopes da Silva 1994, Zhang and Warren 2002) and increases the excitability of NAc neurons by acting on postsynaptic mAChRs (Uchimura and North 1990). In this study, we performed intracellular recordings in rat NAc coronal slice preparation to identify any differential effects of mAChR signaling on synaptic transmission in the dorsal and ventral NAc core.

\section{Materials and Methods}

Animals

Experiments were performed on 3-8 week old Sprague-Dawley rats of male and female. Rats were obtained from the Experimental Animal Center of Zhejiang Province, China. All procedures were approved by the Animal Care and Use Committee of the Institute of Psychology, Chinese Academy of Sciences, and all efforts were made to minimize the rats' suffering and the number of rats used.

\section{NAc slice preparation}

Rat NAc slices were prepared as follows. Rats were anaesthetized with diethyl ether and decapitated, and the brain was quickly transferred to cold $\left(0-4{ }^{\circ} \mathrm{C}\right)$ artificial cerebrospinal fluid (ACSF) of the following composition (in mM): $\mathrm{NaCl} 126, \mathrm{KCl} 2.5, \mathrm{NaH}_{2} \mathrm{PO}_{4} 1.25$, $\mathrm{MgCl}_{2} 1, \mathrm{CaCl}_{2} 2, \mathrm{NaHCO}_{3} 26$, and glucose 20. A tissue block containing the NAc was glued to an agar chunk. Coronal brain slices (350-400 $\mu \mathrm{m}$ thick) containing the
NAc were sectioned by Vibratome 1000 or 1500 series tissue slicers (Technical Products International Inc., USA). Slices were incubated in oxygenated ACSF at room temperature $\left(22-28^{\circ} \mathrm{C}\right)$ for $60 \mathrm{~min}$, after which a single slice was transferred to a recording chamber $(0.5 \mathrm{ml})$. In the recording chamber, the slice was superfused by oxygenated ACSF continuously at room temperature.

\section{Intracellular recordings}

Borosilicate, filament containing capillary glass micropipettes (FSG12, OD: $1.2 \mathrm{~mm}$, ID: $0.6 \mathrm{~mm}$ ) were pulled into sharp glass microelectrodes using a Micropipette Puller (Model P-97, Sutter Instrument Co., USA). Intracellular recordings were performed in NAc core neurons with microelectrodes filled with $3 \mathrm{M}$ potassium acetate (input impedance of 70-120 M $\Omega$ ), using an AxoClamp 2B amplifier (Axon Instrument Inc., USA) in current-clamp mode. The location of the recorded neurons was visualized by a stereo microscope, using the anterior commissure (AC), neostriatum, septum and lateral ventricles as landmarks based on a rat brain stereotaxis atlas (Paxinos and Watson 1986).

Data acquisition was performed with pClamp7.0 software (Axon Instruments) and off-line analysis was performed with Clampfit software (Axon Instruments).

\section{Presynaptic stimulation}

At resting potential, depolarizing synaptic potentials (DPSP) were evoked by focal stimulation with a concentric bipolar tungsten stimulating electrode, placed onto the surface of the slice dorsal or ventral to the AC (D-DPSP and V-DPSP). The distance between the stimulating electrode and the recording electrode was 0.3 $0.5 \mathrm{~mm}$. Electrical stimulation (single square wave, 20$100 \mathrm{~V}, 0.1 \mathrm{~ms}$ duration) was delivered at $0.2 \mathrm{~Hz}$, and stimulus intensity was kept invariant throughout the recording period. Paired-pulse stimulation was delivered with interpulse intervals (IPIs) of 50, 100 and $150 \mathrm{~ms}$, and other stimulation parameters were the same as above. Paired-pulse ratio (PPR) was evaluated as the ratio of the second DPSP amplitude to the first, and was used to distinguish pre- and/or postsynaptic mechanisms.

\section{Drug application}

Drugs were prepared in stock solutions and perfused at set concentrations via a pump. After recording a stable DPSP for at least $15 \mathrm{~min}$, ACSF containing muscarine was superfused for 3-10 min, which was 
sufficient for depression of the synaptic potential to achieve a steady state, and was then followed by washout with control ACSF. Muscarine, 6-cyano-7nitroquinoxaline-2,3-dione (CNQX) and DL-2-amino-5phosphonovaleric acid (APV) were purchased from Sigma-Aldrich Co., USA, and tetrodotoxin (TTX) was purchased from Fisheries Science and Technology Development Company of Hebei Province, China.

\section{Statistics}

All averaged values are expressed as mean \pm SEM. Statistical comparisons were performed using Student's unpaired or paired $t$ test, one-way ANOVA followed by post-hoc Holm-Sidak test, and $p<0.05$ was considered statistically significant.

Table 1. Electrophysiological parameters of NAc core neurons before and during muscarine superfusion.

\begin{tabular}{|c|c|c|c|c|c|}
\hline & \multirow{2}{*}{$\begin{array}{l}\text { Control } \\
(n=22)\end{array}$} & \multicolumn{4}{|c|}{ Muscarine $(\mu \mathrm{M})$} \\
\hline & & $1(n=22)$ & $3(n=21)$ & $10(n=20)$ & $30(n=21)$ \\
\hline$R M P(m V)$ & $-82.9 \pm 1.7$ & $-83.9 \pm 2.0$ & $-80.7 \pm 2.4$ & $-79.5 \pm 1.6$ & $-78.6 \pm 1.6$ \\
\hline Membrane resistance (M $\Omega)$ & $63.9 \pm 4.7$ & $70.2 \pm 5.1$ & $59.1 \pm 6.6$ & $65.8 \pm 4.8$ & $73.6 \pm 6.7$ \\
\hline Spike amplitude (mV) & $58.5 \pm 1.8$ & $58.4 \pm 1.4$ & $56.0 \pm 2.3$ & $57.8 \pm 2.6$ & $52.2 \pm 1.9$ \\
\hline
\end{tabular}

One-way ANOVA: $p>0.05$ in all parameters

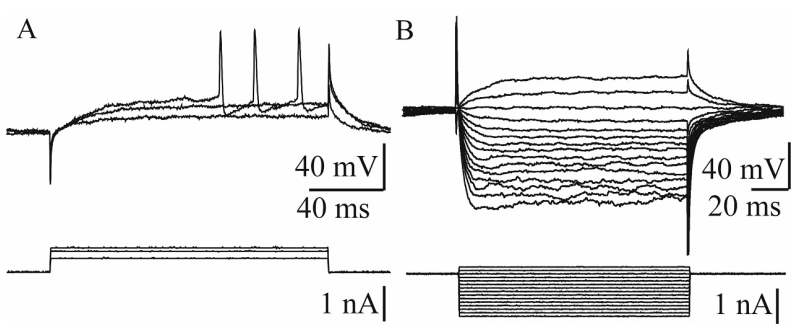

Fig. 1. Typical membrane properties of NAc core neurons, evaluated by recording responses to current steps. (A) The electrotonic membrane potential (top) was obtained with a step of the depolarizing current injected, $0.4 \mathrm{nA}, 0.6 \mathrm{nA}$ and $0.7 \mathrm{nA}$, respectively (bottom), indicating a slowly depolarizing ramp. The resting potential of this neuron was $-78 \mathrm{mV}$. (B) Electrotonic membrane potentials induced by a series of hyperpolarization current steps, in which the most negative one was $-1.2 \mathrm{nA}$ with decrements of current pulses of $0.1 \mathrm{nA}$, showing the inward rectification. The resting potential was $-74 \mathrm{mV}$.

\section{Results}

Effects of muscarine on membrane properties of NAc core neurons

Results were obtained from 53 neurons using intracellular recordings. These neurons generally exhibited electrophysiological characteristics of medium spiny neurons (MSNs), i.e., relatively negative average RMP of $-79.5 \pm 1.0 \mathrm{mV}$, input resistance of $63.4 \pm 2.6 \mathrm{M} \Omega$, spike amplitude of $61.8 \pm 1.1 \mathrm{mV}$, spontaneous spike firing and spontaneous synaptic activity were not observed, a slow depolarizing ramp (Fig. 1A) was observed in more than half of the neurons (29/53) and inward rectification (Fig. 1B) was observed in nearly one third of the neurons (17/53).

After bath application of the selective mAChR agonist muscarine at $1 \mu \mathrm{M}, 3 \mu \mathrm{M}, 10 \mu \mathrm{M}$ and $30 \mu \mathrm{M}$, there was no change in the intrinsic membrane properties of the NAc core neurons in which ventral or dorsal DPSP were evoked, so we pooled them (RMPs: $F_{(4,56)}=1.4$, $p>0.05$; membrane resistance: $\mathrm{F}_{(4,64)}=0.8, p>0.05$; spike amplitude: $\left.\mathrm{F}_{(4,58)}=1.6, p>0.05\right)$ (Table 1).

\section{Muscarine produces a differential dose-dependent} decrease in V-DPSP and D-DPSP

At RMPs, focal electrical stimulation of the ventral and dorsal NAc evoked DPSP in 29 and 34 neurons, respectively, defined as V-DPSP and D-DPSP. The amplitudes of both DPSPs were graded with the intensity of the stimulation (ventral $n=10$, dorsal $n=4$ ) (Fig. 2A), decreased with membrane potential depolarization and increased with membrane potential hyperpolarization (ventral $n=3$, dorsal $n=3$ ) (Fig. 2B). These synaptic potentials were also reversibly abolished by low $\mathrm{Ca}^{2+} /$ high $\mathrm{Mg}^{2+}$ ACSF solution (ventral $\mathrm{n}=3$, dorsal $n=2)$ or by TTX $(0.5 \mu \mathrm{M})($ ventral $n=2$, dorsal $n=3)$ (Fig. 2C). The amplitudes of V-DPSP and D-DPSP were reduced to $15-17 \%$ and $14-25 \%$ of control, respectively, when CNQX $(10 \mu \mathrm{M})$ was applied (ventral $\mathrm{n}=3$, dorsal $\mathrm{n}=2$ ) (Fig. 2D), and there was minimal further reduction when CNQX $(10 \mu \mathrm{M})$ and APV $(30 \mu \mathrm{M})$ were co-applied 
(Fig. 2D). We found no difference between V-DPSP and D-DPSP at the same stimulus range (unpaired $t$ test in all parameters, $p>0.05$ ), and the quantified parameters of both responses are summarized in Table 2. The latencies of V-DPSP and D-DPSP remained constant when the stimulus intensity was changed, indicating that the two types of DPSPs were both monosynaptic.
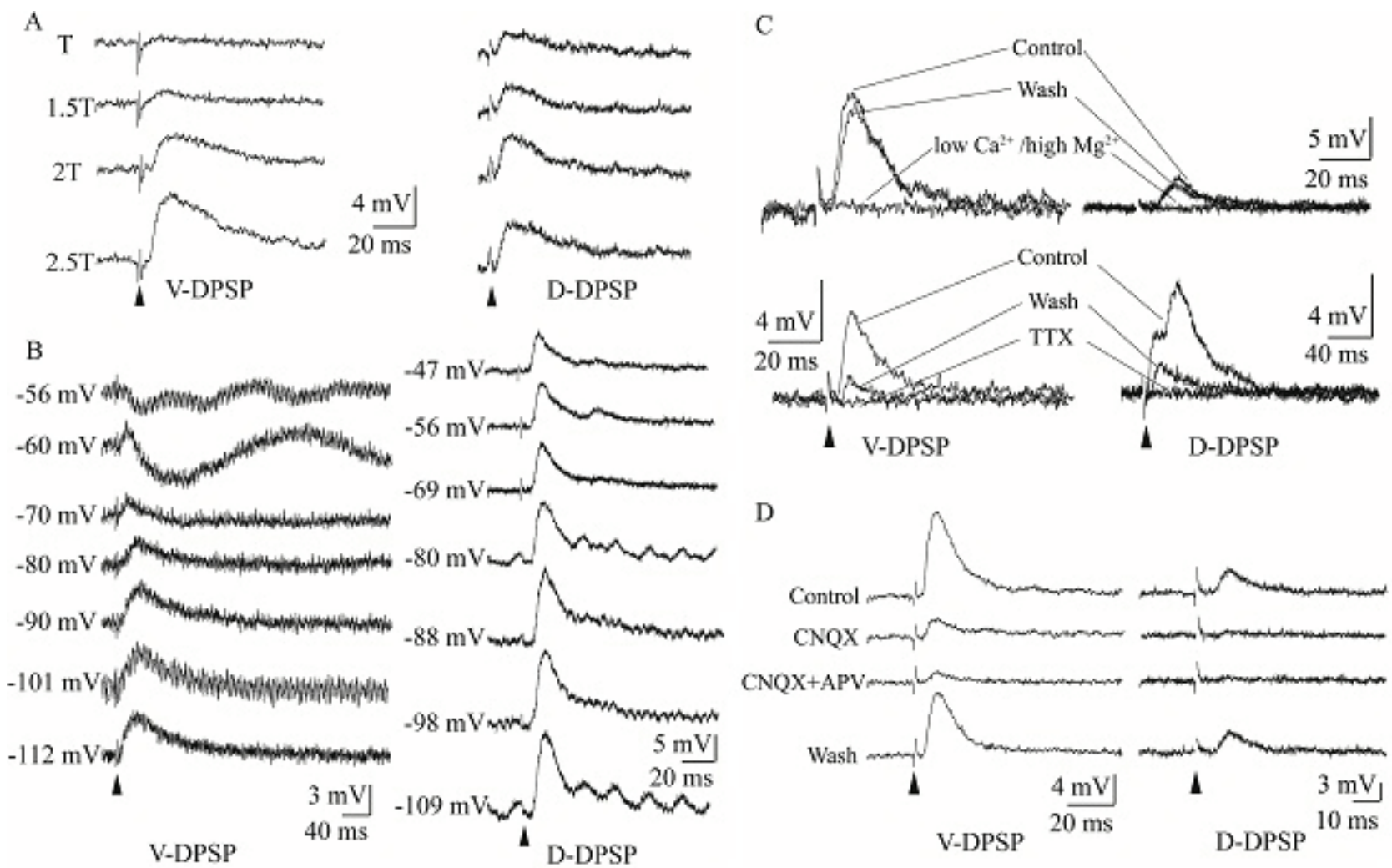

Fig. 2. Cell electrophysiological properties of postsynaptic potentials evoked by ventral and dorsal focal electrical stimuli in NAc core neurons. (A) Both DPSP amplitudes were graded with stimulus intensities. T shows the threshold intensity of stimulus to evoke DPSP. (B) Responses to the same electrical stimulus at different membrane potentials by injecting hyperpolarizing and depolarizing currents into the neurons. The more negative the membrane potential, the greater the DPSP amplitude. (C) The top traces show that both DPSPs were reversibly abolished by low $\mathrm{Ca}^{2+} /$ high $\mathrm{Mg}^{2+}$ ACSF solution, and the bottom traces indicate that both DPSPs were abolished by TTX (0.5 $\left.\mu \mathrm{M}\right)$ with partial recovery. (D) Effect of a quisqualate/kainate receptor antagonist on both DPSPs. $10 \mu \mathrm{M}$ CNQX almost completely abolished the DPSPs, and CNQX superfusion along with APV $(50 \mu \mathrm{M})$ had minimal further inhibitory effect, with recovery on washout.

Table 2. Cell electrophysiological parameters of ventral and dorsal stimulus evoked depolarizing synaptic potentials.

\begin{tabular}{lcc}
\hline & $\begin{array}{c}\text { V-DPSP } \\
(\mathbf{n = 2 9 )}\end{array}$ & $\begin{array}{c}\text { D-DPSP } \\
(\mathbf{n = 3 4 )}\end{array}$ \\
\hline Amplitude $(\mathrm{mV})$ & $6.4 \pm 0.8$ & $8.6 \pm 0.9$ \\
Area under curve $\left(\mathrm{m} V^{*} \mathrm{~ms}\right)$ & $171.1 \pm 29.2$ & $231.6 \pm 31.1$ \\
Duration (ms) & $84.6 \pm 8.3$ & $77.5 \pm 5.3$ \\
Latency $(\mathrm{ms})$ & $4.4 \pm 0.2$ & $4.8 \pm 0.3$ \\
Half-width (ms) & $16.3 \pm 1.6$ & $18.1 \pm 1.5$ \\
Rise time (ms) & $6.1 \pm 0.7$ & $5.8 \pm 0.5$ \\
Decay time (ms) & $37.0 \pm 5.4$ & $38.5 \pm 4.2$ \\
\hline
\end{tabular}

Unpaired $t$ test: $p>0.05$ in all parameters
At RMPs, muscarine attenuated the amplitude of V-DPSP in 18 tested cells in a dose-dependent manner $\left(\mathrm{F}_{(4,53)}=18.2, p<0.05\right)$, with partial recovery after wash. Uniformly, muscarine also reversibly decreased the amplitude of D-DPSP in 14 neurons in a dose-dependent manner $\left(\mathrm{F}_{(4,35)}=30.6, p<0.05\right)$. The areas under the curves of V-DPSP and D-DPSP were also decreased (ventral $\mathrm{F}_{(4,52)}=6.9, \quad p<0.05 ;$ dorsal $\left.\mathrm{F}_{(4,35)}=21.1, p<0.05\right)$. The normalized parameters of both responses are summarized in Table 3. The time course of V-DPSP, evaluated by duration, half-width, rise time and decay time, were not altered during superfusion of muscarine at all concentrations except at $30 \mu \mathrm{M}$ relative to control. However, the duration, half-width and decay time of DDPSP were shortened compared to control with application of $30 \mu \mathrm{M}$ muscarine (paired $t$ test, $p<0.05$ ). 
Table 3. Comparisons of concentration-dependent inhibition of muscarine on V-DPSP and D-DPSP.

\begin{tabular}{|c|c|c|c|c|}
\hline & \multicolumn{2}{|c|}{ Amplitude (\%) } & \multicolumn{2}{|c|}{ Area under curve (\%) } \\
\hline & V-DPSP & D-DPSP & V-DPSP & D-DPSP \\
\hline $1 \mu M$ & $72.1 \pm 6.5 *(10)$ & $73.0 \pm 7.0 *(6)$ & $73.7 \pm 11.2(10)$ & $64.9 \pm 6.0 *(6)$ \\
\hline $3 \mu M$ & $63.0 \pm 11.5 *(7)$ & $53.3 \pm 6.0 *(6)$ & $60.6 \pm 14.9 *(7)$ & $47.9 \pm 8.6 *(6)$ \\
\hline $10 \mu M$ & $61.1 \pm 4.6^{*}(13)$ & $52.3 \pm 7.2 *(9)$ & $62.0 \pm 7.4 *(13)$ & $46.1 \pm 11.9^{*}(9)$ \\
\hline $30 \mu M$ & $52.6 \pm 4.3 *(9)$ & $32.4 \pm 5.4 *(5)$ & $54.4 \pm 9.2 *(9)$ & $18.0 \pm 4.2 *(5)$ \\
\hline
\end{tabular}

One-way ANOVA followed by post-hoc Holm-Sidak test, $* p<0.05$ vs. control

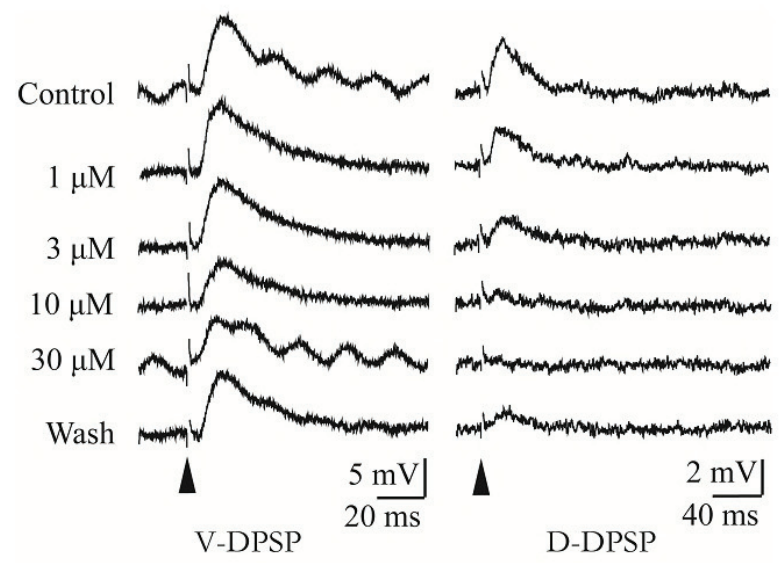

D-DPSP

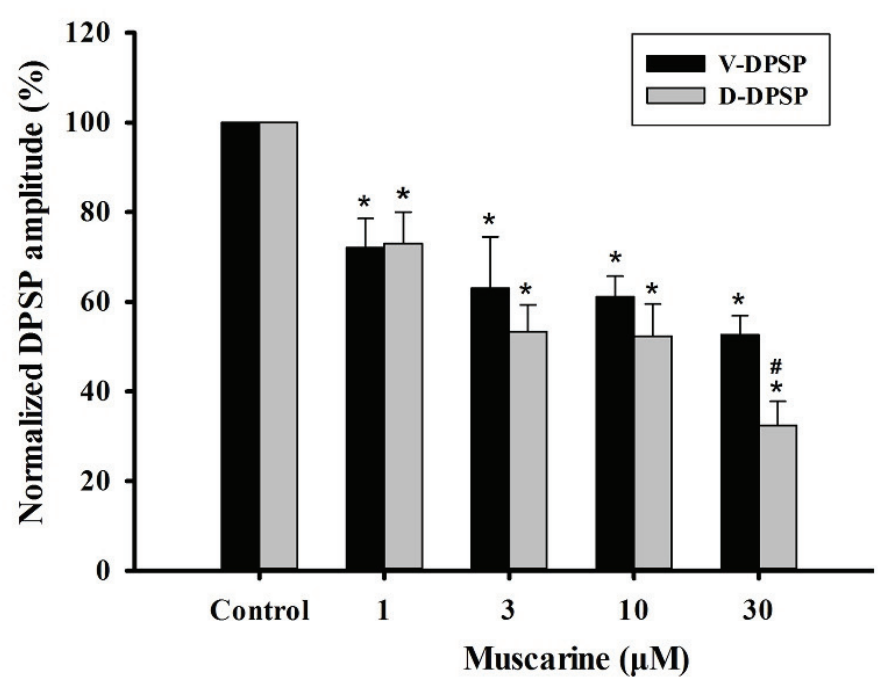

Fig. 3. Muscarine inhibited V-DPSP and D-DPSP to a different degree. Left, representative DPSPs obtained from ventral and dorsal focal electrical stimuli were depressed by muscarine at several concentrations. Arrow head indicates the electrical stimulus artifact. Right, summary of normalized V-DPSP (black) and D-DPSP (gray) amplitudes at several concentrations of muscarine. Bars represent mean proportions $( \pm \mathrm{SEM})$ determined in two independent experiments. $* p<0.05$ compared with untreated control (one-way ANOVA followed by the Holm-Sidak test). Muscarine elicited a stronger inhibitory effect on D-DPSP amplitude than V-DPSP amplitude, \# $p<0.05$ compared with V-DPSP (unpaired $t$ test).

We also observed that the inhibition ratio of DDPSP amplitude and area under curve by muscarine at $30 \mu \mathrm{M}$ was greater than that of V-DPSP (unpaired $t$ test, $p<0.05$ ), but not at lower concentrations of muscarine (Fig. 3).

Muscarine increases the PPR of V-DPSPs and D-DPSPS

Paired-pulse facilitation of ventral or dorsal focal electrical stimulation evoked DPSP was observed in most of the tested neurons when IPIs was $50 \mathrm{~ms}, 100 \mathrm{~ms}$ and $150 \mathrm{~ms}$. The PPR of V-DPSPs (i.e. V-PPR) and DDPSPs (i.e. D-PPR) were both decreased as the IPIs extended (ventral $\mathrm{n}=13, p<0.05$; dorsal $\mathrm{n}=21, p<0.05$ ) (Fig. 4A). V-PPR was $1.4 \pm 0.1,1.2 \pm 0.1$ and $1.1 \pm 0.1$ at IPIs $50 \mathrm{~ms}, 100 \mathrm{~ms}$ and $150 \mathrm{~ms}$, respectively, while DPPR was $1.6 \pm 0.1,1.5 \pm 0.1$ and $1.4 \pm 0.1$. As shown in Figure 4A, D-PPR was statistically greater than V-PPR (unpaired $t$ test, $p<0.05$ ) at $100 \mathrm{~ms}$ and $150 \mathrm{~ms}$ intervals.

To assess whether presynaptic mechanisms were involved in the muscarinic inhibition of DPSP, V-DPSPs and D-DPSPs evoked by paired-pulse stimulation at $100 \mathrm{~ms}$ intervals were examined before and after muscarine superfusion. The PPR of V-DPSPs and DDPSPs was increased in most neurons examined (paired $t$ test, ventral $n=6$, dorsal $n=3, p<0.05$ ) (Fig. 4B).

\section{Discussion}

In this study, we investigated the effects of the mAChR agonist muscarine on the DPSP and PPR evoked by ventral or dorsal local circuits in NAc core. We found that muscarine elicited a reduction of both dorsal and ventral DPSP but had no influence on membrane properties of the NAc core neurons. Moreover, we found 
that the inhibitory effect on D-DPSP was significantly larger than that on V-DPSP at high muscarine concentration $(30 \mu \mathrm{M})$. Furthermore, muscarine increased both D-PPR and V-PPR. Our results suggest the existence of distinct dorsal and ventral subregions in the NAc core that are differentially modulated by muscarine.
A

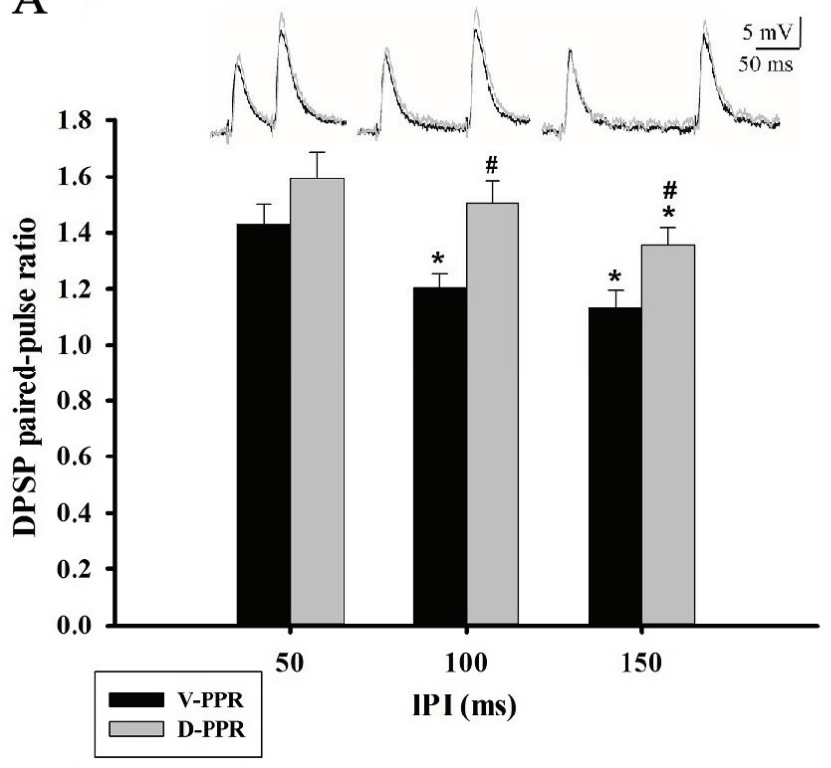

B

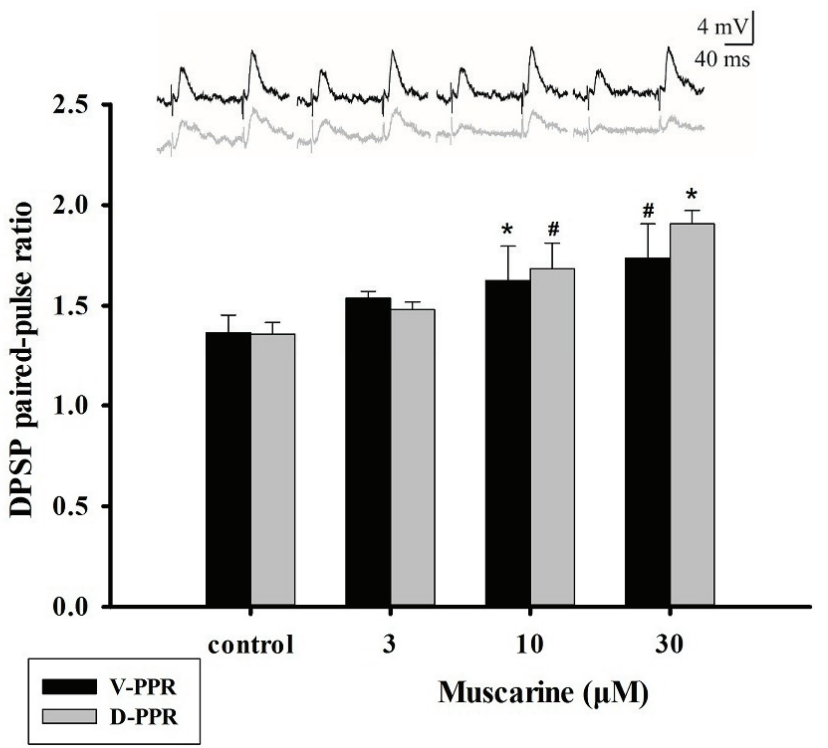

Fig. 4. PPR was increased by muscarine. (A) paired-pulse facilitation was induced by paired-pulse stimulation at different IPIs. V-PPR (black, $n=13$ ) and D-PPR (gray, $n=21$ ) were decreased along with the increase of IPIs. D-PPR was greater than V-PPR. * $p<0.05$ compared with $50 \mathrm{~ms}$ (paired $t$ test). \# $p<0.05$ compared with V-PPR (unpaired $t$ test). Inset shows the representative paired-pulse facilitation evoked from ventral and dorsal focal electrical stimuli (ventral, black; dorsal, gray). (B) summary of V-PPR (black) and D-PPR (gray) at several concentration of muscarine. Error bars represent mean proportions $( \pm \mathrm{SEM})$. $* p<0.05 ; \# p=0.07$ compared with untreated control (paired $t$ test).

Neuroanatomical studies have suggested that the main origin of glutamatergic afferent fibers traversing the ventral and dorsal NAc is separate (Groenewegen et al. 1999, Humphries and Prescott 2010, Voorn et al. 2004). In the present study, both V-DPSP and D-DPSP were mainly mediated by AMPA/KA receptors (Fig. 2) and showed similar electrophysiological characteristics. These data were in agreement with previous reports (O'Donnell and Grace 1993, Pennartz et al. 1991, Pennartz and Lopes da Silva 1994, Uchimura et al. 1989). Moreover, we found that D-PPR was greater than V-PPR (Fig. 4A), suggesting that glutamatergic afferent properties are different between dorsal and ventral afferent fibers within the NAc core. The specific pathways of the ventral and dorsal NAc core await further examination.

Axonal arborization originating from cholinergic interneurons is distributed throughout the NAc core, and ACh plays critical roles in the functional regulation of NAc core neurons via the activation of mAChRs. Muscarine has been used as a specific agonist of mAChRs in many brain regions including the superior colliculus, dorsal striatum, suprachiasmatic nucleus and hippocampus (Lin et al. 2004, Sooksawate and Isa 2006, Vogt and Regehr 2001, Yang et al. 2010). In agreement with previous studies (Pennartz and Lopes da Silva 1994, Sugita et al. 1991, Zhang and Warren 2002), we found that muscarine inhibits V-DPSP and D-DPSP in a dosedependent manner. This suppressive effect of muscarine on DPSP may be mainly via presynaptic mechanisms. Firstly, PPR were increased after bath application of muscarine, indicating that muscarine likely decreases the probability of glutamatergic transmitter release (Schulz 1997, Stuber et al. 2011). Secondly, muscarine depressed DPSP but did not change the RMPs or the membrane input resistance, which rules out the possibility of somatic and proximal dendritic activity.

Considering that the effectiveness of bioactive substances and neuromodulators might vary depending on the nature and state of the neural network in which they operate (Pennartz and Lopes da Silva 1994), our results showing that the inhibitory effect of $30 \mu \mathrm{M}$ muscarine in D-DPSP amplitude and area under the curve was greater than that in V-DPSP suggests the dorsal and 
ventral NAc core are differentially innervated by glutamatergic afferents and exhibit different modulation by muscarine. The density or amount of muscarinic receptors located on presynaptic glutamate terminals in dorsal afferents might be higher than that in ventral afferents, since muscarine attenuated DPSP mainly by decreasing glutamatergic transmitter release (Pennartz and Lopes da Silva 1994, Zhang and Warren 2002). Supporting this hypothesis, similar phenomena have been found in anatomical studies showing that the distributions of muscarinic receptors are different between the NAc core and shell (Wirtshafter and Osborn 2004). Moreover, behavioral studies suggest that the roles of muscarinic receptors in the NAc core and shell differ, as muscarinic receptors in NAc shell modulate drug seeking selectively whereas those receptors in the NAc core modulate both food and drug seeking (Yee et al. 2011). We note that intrinsic postsynaptic neural elements such as distal dendritic inhibitory transmission shunt cannot be excluded, since the reduction of D-DPSP time course was contingent upon the attenuation of its amplitude. With the knowledge that glutamatergic striatal afferents are topographically organized, the findings from this study further clarify how these subregions are anatomically and functionally modulated in the NAc core.

In conclusion, the present data indicate that the activation of $\mathrm{mAChRs}$ by muscarine decreases excitatory neurotransmission of the dorsal and ventral pathway in the NAc core to different degrees, and this effect might be mediated by presynaptic mechanisms. Given that the NAc core is implicated in motivated behaviors, our characterization of structural and functional subdivisions of the NAc core might clarify different behavioral contributions of these regions.

\section{Conflict of Interest}

There is no conflict of interest.

\section{Acknowledgements}

The study was supported by the Chinese Academy of Sciences grants (KSCX2-EW-R-12), the National Basic Research Program grants (2009CB522002), the National Natural Science Foundation grants (31170988), Key Laboratory of Mental Health, Institute of Psychology, CAS and the Anhui Provincial Natural Science Foundation, China (090413084, WMY). We thank Dr. Yong-Hui Li, Dr. Eiman Azim and Dr. Ning Zhu for help in revision of the manuscript.

\section{References}

BERRIDGE KC: The debate over dopamine's role in reward: the case for incentive salience. Psychopharmacology (Berl) 191: 391-431, 2007.

BRITT JP, BENALIOUAD F, MCDEVITT RA, STUBER GD, WISE RA, BONCI A: Synaptic and behavioral profile of multiple glutamatergic inputs to the nucleus accumbens. Neuron 76: 790-803, 2012.

CRESPO JA, STURM K, SARIA A, ZERNIG G: Activation of muscarinic and nicotinic acetylcholine receptors in the nucleus accumbens core is necessary for the acquisition of drug reinforcement. $J$ Neurosci 26: 6004-6010, 2006.

GOTO Y, GRACE AA: Dopaminergic modulation of limbic and cortical drive of nucleus accumbens in goal-directed behavior. Nat Neurosci 8: 805-812, 2005.

GROENEWEGEN HJ, WRIGHT CI, BEIJER AV, VOORN P: Convergence and segregation of ventral striatal inputs and outputs. Ann N Y Acad Sci 877: 49-63, 1999.

HIKIDA T, KITABATAKE Y, PASTAN I, NAKANISHI S: Acetylcholine enhancement in the nucleus accumbens prevents addictive behaviors of cocaine and morphine. Proc Natl Acad Sci U S A 100: 6169-6173, 2003.

HUMPHRIES MD, PRESCOTT TJ: The ventral basal ganglia, a selection mechanism at the crossroads of space, strategy, and reward. Prog Neurobiol 90: 385-417, 2010.

KALIVAS PW, LALUMIERE RT, KNACKSTEDT L, SHEN H: Glutamate transmission in addiction. Neuropharmacology 56 (Suppl 1): 169-173, 2009.

KELLEY AE: Ventral striatal control of appetitive motivation: role in ingestive behavior and reward-related learning. Neurosci Biobehav Rev 27: 765-776, 2004.

LALUMIERE RT, KALIVAS PW: Glutamate release in the nucleus accumbens core is necessary for heroin seeking. J Neurosci 28: 3170-3177, 2008. 
LIN JY, CHUNG KKH, DE CASTRO D, FUNK GD, LIPSKI J: Effects of muscarinic acetylcholine receptor activation on membrane currents and intracellular messengers in medium spiny neurones of the rat striatum. Eur $J$ Neurosci 20: 1219-1230, 2004.

MEREDITH GE, BALDO BA, ANDREZJEWSKI ME, KELLEY AE: The structural basis for mapping behavior onto the ventral striatum and its subdivisions. Brain Struct Funct 213: 17-27, 2008.

O'DONNELL P, GRACE AA: Physiological and morphological properties of accumbens core and shell neurons recorded in vitro. Synapse 13: 135-160, 1993.

PAXINOS G, WATSON C: The Rat Brain in Stereotaxic Coordinates. Academic Press, Sydney, 1986.

PENNARTZ CM, LOPES DA SILVA FH: Muscarinic modulation of synaptic transmission in slices of the rat ventral striatum is dependent on the frequency of afferent stimulation. Brain Res 645: 231-239, 1994.

PENNARTZ CM, BOEIJINGA PH, KITAI ST, LOPES DA SILVA FH: Contribution of NMDA receptors to postsynaptic potentials and paired-pulse facilitation in identified neurons of the rat nucleus accumbens in vitro. Exp Brain Res 86: 190-198, 1991.

PRATT WE, KELLEY AE: Nucleus accumbens acetylcholine regulates appetitive learning and motivation for food via activation of muscarinic receptors. Behav Neurosci 118: 730-739, 2004.

SCHULZ PE: Long-term potentiation involves increases in the probability of neurotransmitter release. Proc Natl Acad Sci U S A 94: 5888-5893, 1997.

SHIRAYAMA Y, CHAKI S: Neurochemistry of the nucleus accumbens and its relevance to depression and antidepressant action in rodents. Curr Neuropharmacol 4: 277-291, 2006.

SOOKSAWATE T, ISA T: Properties of cholinergic responses in neurons in the intermediate grey layer of rat superior colliculus. Eur J Neurosci 24: 3096-3108, 2006.

STUBER GD, SPARTA DR, STAMATAKIS AM, VAN LEEUWEN WA, HARDJOPRAJITNO JE, CHO S, TYE KM, KEMPADOO KA, ZHANG F, DEISSEROTH K, BONCI A: Excitatory transmission from the amygdala to nucleus accumbens facilitates reward seeking. Nature 475: 377-380, 2011.

STUBER GD, BRITT JP, BONCI A: Optogenetic modulation of neural circuits that underlie reward seeking. Biol Psychiatry 71: 1061-1067, 2012.

SUGITA S, UCHIMURA N, JIANG ZG, NORTH RA: Distinct muscarinic receptors inhibit release of gammaaminobutyric acid and excitatory amino acids in mammalian brain. Proc Natl Acad Sci U S A 88: 2608-2611, 1991.

UCHIMURA N, NORTH RA: Muscarine reduces inwardly rectifying potassium conductance in rat nucleus accumbens neurones. J Physiol 422: 369-380, 1990.

UCHIMURA N, HIGASHI H, NISHI S: Membrane properties and synaptic responses of the guinea pig nucleus accumbens neurons in vitro. $J$ Neurophysiol 61: 769-779, 1989.

VOGT KE, REGEHR WG: Cholinergic modulation of excitatory synaptic transmission in the CA3 area of the hippocampus. J Neurosci 21: 75-83, 2001.

VOORN P, VANDERSCHUREN LJ, GROENEWEGEN HJ, ROBBINS TW, PENNARTZ CM: Putting a spin on the dorsal-ventral divide of the striatum. Trends Neurosci 27: 468-474, 2004.

WIRTSHAFTER D, OSBORN CV: The distribution of $\mathrm{m} 4$ muscarinic acetylcholine receptors in the islands of Calleja and striatum of rats and cynomolgus monkeys. J Chem Neuroanat 28: 107-116, 2004.

YANG JJ, WANG YT, CHENG PC, KUO YJ, HUANG RC: Cholinergic modulation of neuronal excitability in the rat suprachiasmatic nucleus. J Neurophysiol 103: 1397-1409, 2010.

YEE J, FAMOUS KR, HOPKINS TJ, MCMULLEN MC, PIERCE RC, SCHMIDT HD: Muscarinic acetylcholine receptors in the nucleus accumbens core and shell contribute to cocaine priming-induced reinstatement of drug seeking. Eur J Pharmacol 650: 596-604, 2011.

YUAN XR, MADAMBA S, SIGGINS GR: Opioid peptides reduce synaptic transmission in the nucleus accumbens. Neurosci Lett 134: 223-228, 1992.

ZAHM DS: Functional-anatomical implications of the nucleus accumbens core and shell subterritories. Ann NY Acad Sci 877: 113-128, 1999.

ZHANG L, WARREN RA: Muscarinic and nicotinic presynaptic modulation of EPSCs in the nucleus accumbens during postnatal development. J Neurophysiol 88: 3315-3330, 2002. 